\title{
STUDY ON WEB-BASED TOOL FOR REGIONAL AGRICULTURE INDUSTRY STRUCTURE OPTIMIZATION USING AJAX
}

\author{
Xiaodong Huang ${ }^{1}$, Yeping Zhu ${ }^{1, *}$ \\ 1 Agricultural Information Institute of CAAS, Beijing 100081, China; \\ * Corresponding author, Address: Agricultural Information Institute of CAAS, 12 South \\ ZhongGuanChun Road Beijing 100081, China, Tel:+86-10-68919652, Fax:+86-10- \\ 68919652,Email: zhuyp@mail.caas.net.cn
}

\begin{abstract}
According to the research status of regional agriculture industry structure adjustment information system and the current development of information technology, this paper takes web-based regional agriculture industry structure optimization tool as research target. This paper introduces Ajax technology and related application frameworks to build an auxiliary toolkit of decision support system for agricultural policy maker and economy researcher. The toolkit includes a "one page" style component of regional agriculture industry structure optimization which provides agile arguments setting method that enables applying sensitivity analysis and usage of data and comparative advantage analysis result, and a component that can solve the linear programming model and its dual problem by simplex method.
\end{abstract}

Keywords: Agriculture, Industry structure optimize, AJAX, Linear programming

\section{INTRODUCTION}

China is a large agricultural country, and now she has enter a new era of agriculture industry structure strategic adjustment that composed of improving the quality of agricultural products, optimizing the agriculture structure and increasing the income of farmers(Soft Science Committee on the Ministry of Agriculture Research Group. 2000).

Please use the following format when citing this chapter:

Huang, X. and Zhu, Y., 2009, in IFIP International Federation for Information Processing, Volume 295, Computer and Computing Technologies in Agriculture II, Volume 3, eds. D. Li, Z. Chunjiang, (Boston: Springer), pp. 1543-1550. 
Many researchers have studied the application of regional agriculture industry structure adjustment theory, and have built a certain number of information system or decision support system to support this kind of adjustment. By study on these researches, we have found a weakness in those information systems. Many of these information systems include agriculture industry structure optimizing modeling tools. These tools have a common character that they all based on external tools such as MATLAB or SPSS (Xiong. 2006). This character makes a separation between information system and modeling tool that the modeling tool can't make use of data in information system. To address this issue, this paper proposed a solution that makes use of AJAX technology, builds a flexible web-based tool for regional agriculture industry structure optimization modeling and solving.

\section{AJAX TECHNOLOGY AND ASP.NET AJAX FRAMEWORK}

AJAX stands for Asynchronous JavaScript and XML and includes a large set of technologies such as DOM, XMLHttpRequest, XML, XSLT, JSON etc (Garrett. 2005). AJAX technology has been widely implemented in many web applications since it is first brought out in 2005, and it has triggered the revolution of web application that so called trend of Web2.0, and is now supported by many industry giants such as Google and Microsoft.

AJAX brings a new interaction mode between users and web applications which is called asynchronous mode. In a tradition interaction mode, user need to submit each action to web server and wait the page reloaded to see the result. Ajax overcomes the page loading requirements of HTML/HTTPmediated web pages and creates the necessary initial conditions for the evolution of complex, intuitive, dynamic, data-centric user interfaces in web pages. Fig. 1 shows the working mechanism of AJAX:

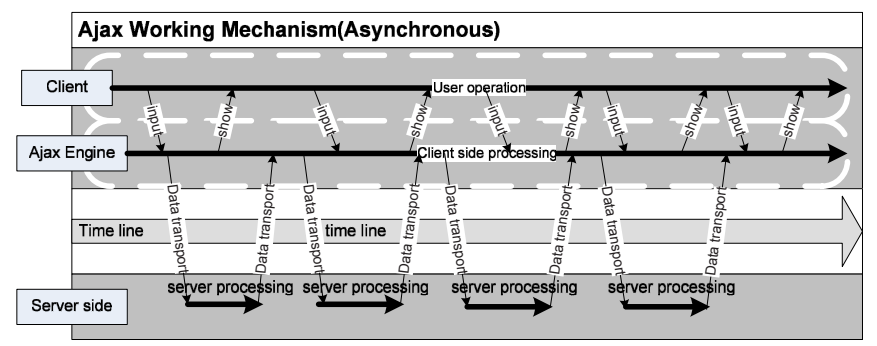

Figure 1. Working Mechanism of AJAX

In this paper, we use ASP.NET AJAX framework as foundation of our web-based tool. ASP.NET AJAX is the name of the solution that provided 
by Microsoft. It is the most powerful and most completely encapsulation of AJAX techniques currently, and provide full support of client side object oriented programming, a large number of client side and server side components, web service integration and ASP.NET components extension.

\section{LINEAR PROGRAMMING FOR REGIONAL AGRICULTURE INDUSTRY STRUCTURE OPTIMIZATION}

According to regional agriculture industry structure adjustment theory, there are two ways to modeling agriculture industry structure optimization problem, one is linear programming, and another is non-linear programming. Linear programming is most used method. Here is the common form of a linear programming model (Hillier et al. 1999):

$$
\operatorname{Max}(\operatorname{Min}) Z=\sum_{i=1}^{n} c_{i} x_{i}
$$

Subject to

$$
\begin{aligned}
& a_{11} x_{1}+a_{12} x_{2}+\cdots+a_{1 n} x_{n} \leq(=, \geq) b_{1} \\
& a_{21} x_{1}+a_{22} x_{2}+\cdots+a_{2 n} x_{n} \leq(=, \geq) b_{2} \\
& \cdots \quad \\
& \quad \begin{array}{l}
\cdots \\
a_{m 1} x_{1}+a_{m 2} x_{2}+\cdots+a_{m n} x_{n} \leq(=, \geq) b_{m} \\
x_{1} \geq 0, x_{2} \geq 0, x_{3} \geq 0 \cdots x_{n} \geq 0
\end{array}
\end{aligned}
$$

This model includes one objective function, several decision variables, and several constraint functions. By research of a large number of empirical study cases, we found that the objective function of the regional agriculture industry structure optimization model often refers to maximize the agricultural economy benefits, or maximize the social benefits, or maximize the environment benefits. We also found the linear programming model mainly includes two type constraint functions, one is constraint that reflects resource restrictions, such as farm land, labors, agriculture machines, and another is constraint that shows the target or objective which need to be achieved, such as security of food, keep advantage and etc.

By summarize the characteristic of regional agriculture industry structure optimization model, we found that the modeling tool is composed of some basic element block. The objective function contains a type and a multimonomial, the constraint function contains a multi-monomial and a type and a right hand side coefficient. Each multi-monomial contains several 
monomials which composed of one coefficient and one decision variable. Because of actual meaning of objective function and constraint functions, coefficients in model objective function and constraint functions often come from agricultural economic data. So the modeling tool can be built on a certain set of agriculture economic data and make use of them.

\section{USER INTERFACE OF MODELING TOOL}

According to the AJAX design principles, the modeling tool is designed as a "one page" style application (Mahemoff et al. 2005). A "one page" style application means user can perform all actions that application provides in one user interface, just like Word or Excel. User can create a model then edit it, save it, and edit it again. With this kind of web application, we can provide the same user experience as desktop application. Fig. 2 shows the user interface of regional agriculture industry structure optimization modeling tool:

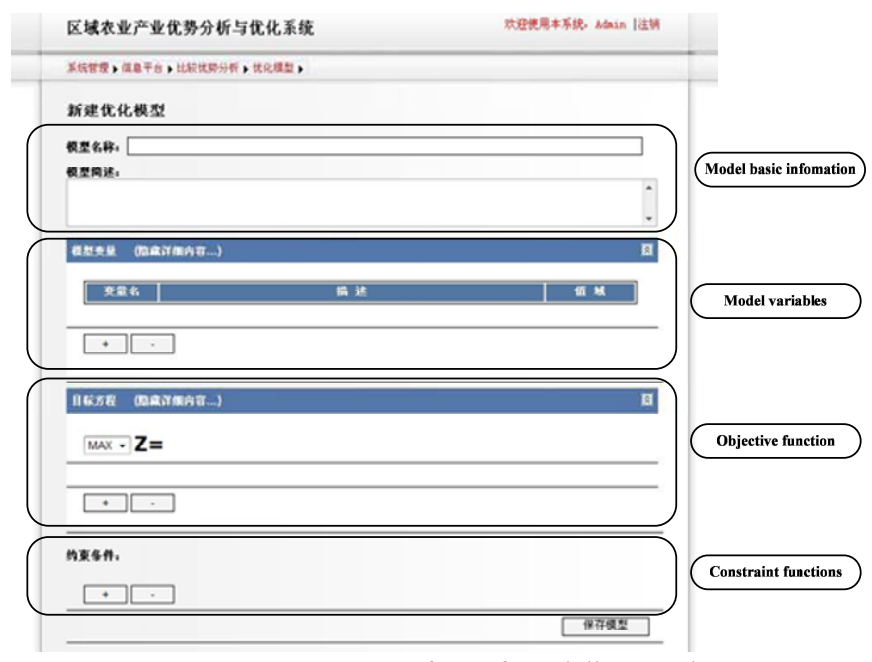

Figure 2. User Interface of Modeling Tool

The whole page is divided to 4 parts; first one is for filling model's basic information such as model name, model description; second part is variables setting panel, user can add or remove decision variable in this part; third part is objective function editing panel; and the last part is for editing constraint functions. The last three parts each contains an add button and a remove button, respectively response for adding or removing element in model. Each part with a title bar can be collapsed by clicking the title bar in order to save the work space and prevent come out with a long page. 
A monomial is designed as a single widget, by clicking the add button in objective function editing panel or constraint function editing panel, user can add a monomial to target function like in fig.3:

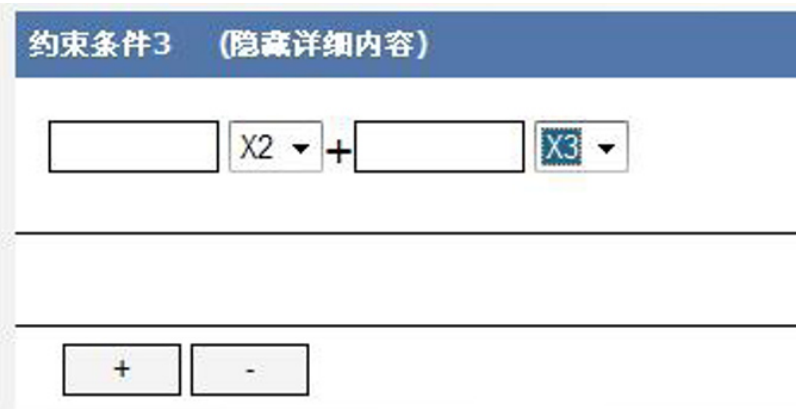

Figure 3. Add a Monomial to Target Function

In order to provide flexibility and make full use of agriculture data and comparative advantage analysis result, regional agriculture industry structure optimization modeling tool provide four ways to set the coefficient of monomial:

- Const coefficient: user input the coefficient with a number when create model, and this number stays unchanged during model calculation.

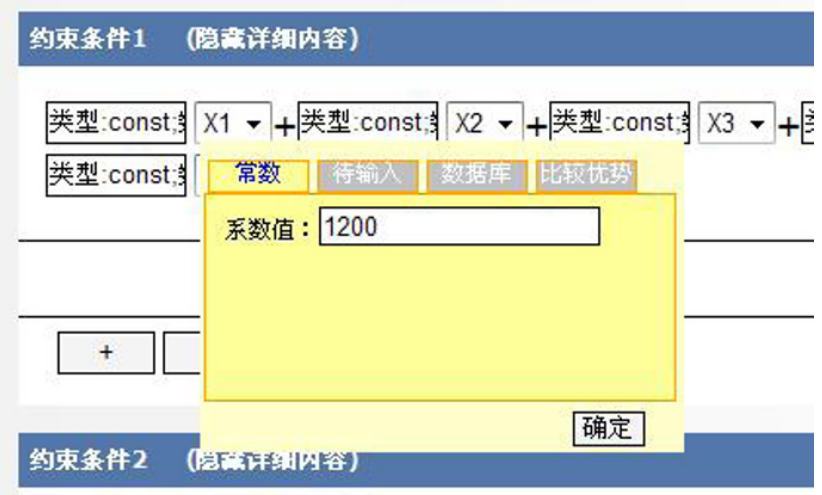

Figure 4. Model Coefficient Setting-Const Coefficients

- Input coefficient: user provides a description of this coefficient, the modeling tool will automatically generate an input textbox before model calculation, and user can input different value of this coefficient in every other calculation. 


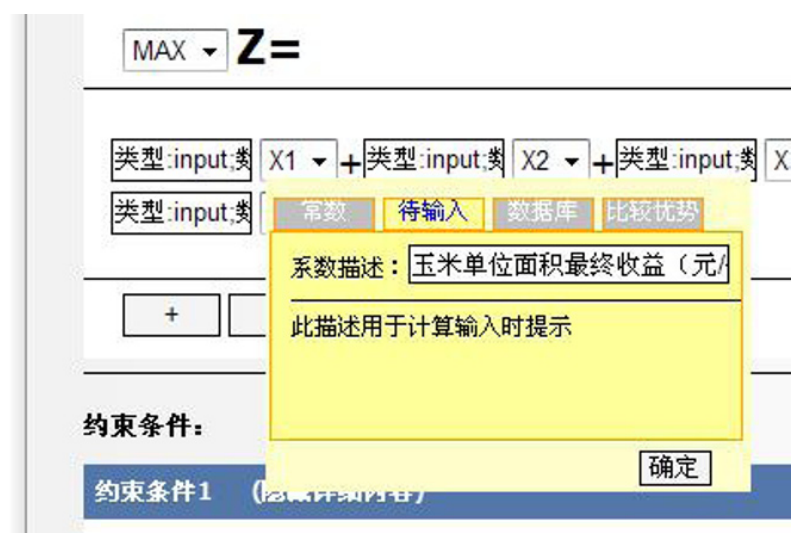

Figure 5. Model Coefficient Setting-Input Coefficients

- From database coefficient: user can set some criteria to locate a data in database, modeling tool will get the actual data when model calculation.

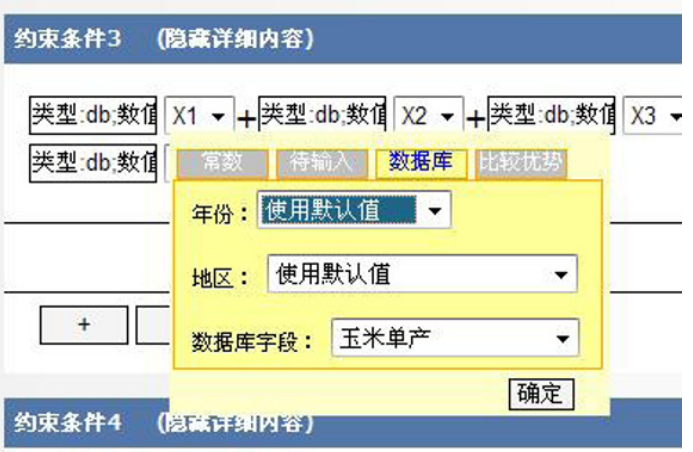

Figure 6. Model Coefficient Setting-From Database Coefficients

- Compare index coefficient: user provides some criteria to find a compare index in the result of comparative advantage analysis, modeling tool will get the actual data when model calculation.

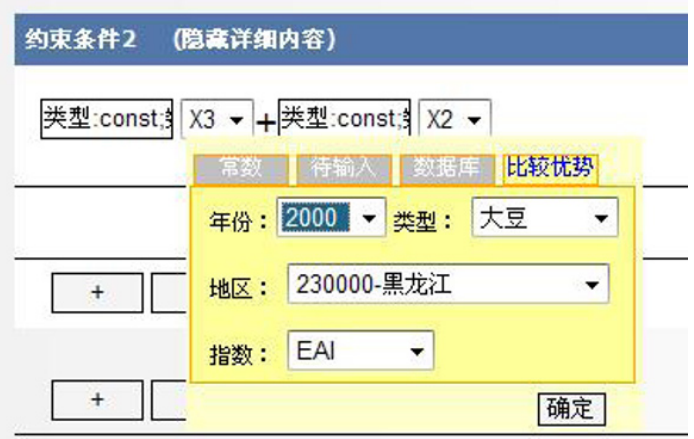

Figure 7. Model Coefficient Setting-Compare Index Coefficients 


\section{COMPONENTS DESIGN OF REGIONAL AGRICULTURE INDUSTRY STRUCTURE OPTIMIZATION MODELING TOOL}

We design the components of regional agriculture industry structure optimization modeling tool according to the N-tiers design principle (Wikipedia. 2007), fig.8 shows the structure of components:

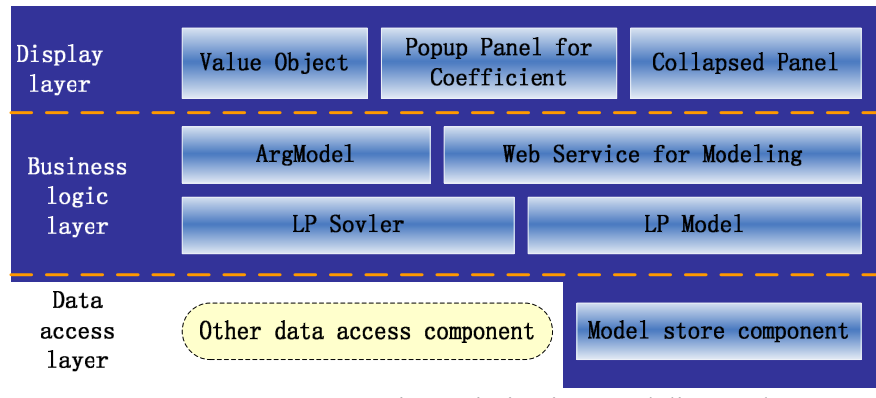

Figure 8. Components in Optimization Modeling tool

- Value object: Value object is used for transporting complex data type between client-side JavaScript and server-side component (Gibbs et al. 2007) ASP.NET AJAX framework can perform this transform automatically.

- Popup panel for coefficient: used for setting coefficient type and related criteria and value. This panel is design according to the popup pattern (Popup pattern. 2006), the popup pattern is one of the most important AJAX design pattern which allows user perform a quick task without distracting from the main flow. In this modeling tool, popup panel allows user set the coefficient without distracting from model building.

- Collapsed panel: after editing a block of model, user can make that block collapse, this action makes higher usability of page space

- Web services for modeling: a set of web services that response directly to client-side requests and dispatch those requests to components in business logic layer.

- ArgModel: a ArgModel object stores all information that user entered in model creation, includes model name, model description, variables name and description, every coefficient's value or find criteria. ArgModel also provides method that returns a LPModel object.

- LPModel: represents a linear programming model that generated from ArgModel. LPModel just contains data and function, and has nothing to do with actual meaning of the model.

- LPSovler: provide algorithm that can solve the linear programming problem and its dual problem by simplex method.

- Model store component: responsible for reading ArgModel information 
from and writing to xml files.

\section{DISCUSSION}

The AJAX technology can bring web application a better user experience and make use of client-side computing power. In this paper, we design and implement a flexible web-based tool for regional agriculture industry structure optimization modeling and solving by using AJAX technology. This modeling tool is a relatively independent component that can be use in agricultural information system, provides linear programming model building, solving abilities.

\section{ACKNOWLEDGEMENTS}

The work described in this paper was funded by National Scientific and Technical Supporting Programs Funded by Ministry of Science and Technology of China (2006BAD10A06), Beijing Municipal Natural Science Foundation (4042026), and Special Fund of Basic Scientific Research and Operation Foundation for Commonweal Scientific Research Institutes (2008J-1-06).

\section{REFERENCES}

Frederick S. Hillier, Gerald J. Lieberman. Introduction to Operation Research(sixth edition). China Machine Press. 1999 http://www.adaptivepath.com/ideas/essays/archives/000385.php

Jesse James Garrett.A New Approach to Web Applications.

Mahemoff, Michael. Ajax Design Patterns. Oreilly \& Associates Inc. 2005

Matt Gibbs, Dan Wahlin. Professional ASP.NET 2.0 AJAX. Wrox Press. 2007

Popup pattern. http://ajaxpatterns.org/Popup

Soft Science Committee on the Ministry of Agriculture Research Group. New Stage of China Agriculture Development. 2000

Wikipedia http://en.wikipedia.org/wiki/Multitier_architecture

Xiong Shuanglin. A Research on Decision Support System for Agriculture Structure Adjustment at County Level--A Case Study in Hua County of Henan Province. Chinese Academy of Agricultural Sciences. 2006 